\title{
Hacia una reducción del contenido de cobre en pinturas antiincrustantes
}

\author{
Mónica Teresita García ${ }^{1}$, Miriam Cristina Pérez ${ }^{1,2}$, \\ Mirta Elena Stupak ${ }^{1}$, Guillermo Blustein ${ }^{1,2}$
}

\author{
${ }^{1}$ Centro de Investigación y Desarrollo en Tecnología de Pinturas, 52 e/ 121 y 122 La Plata (1900), Argentina \\ e-mail: biofouling@ cidepint.gov.ar \\ ${ }^{2}$ Universidad Nacional de La Plata, La Plata (1900), Argentina
}

\section{RESUMEN}

El biofouling conduce al deterioro de las estructuras emplazadas en el mar acarreando graves pérdidas económicas. La aplicación de pinturas a base de óxido cuproso es el método más difundido para el control de las incrustaciones aún siendo nocivas para los ecosistemas marinos. Ante la necesidad de desarrollar formulaciones más inocuas se propone el uso de productos de origen natural que puedan reemplazar parte del cobre disminuyendo su aporte al medio ambiente.

El timol se aísla de los aceites esenciales de numerosas plantas (tomillo, orégano, albahaca) y ha sido ampliamente estudiado por sus propiedades antimicrobianas. La hipótesis que se plantea es que inhibiría el proceso inicial del asentamiento de organismos incrustantes.

El objetivo de este trabajo es reducir el contenido de cobre en las pinturas antifouling empleando al timol como aditivo.

Se prepararon dos pinturas antifouling, una conteniendo $16 \% \mathrm{v} / \mathrm{v}$ de cobre y otra con $1,6 \%$ de cobre $+2 \%$ de timol. Las pinturas se aplicaron sobre paneles de acrílico y se sumergieron en el puerto de Mar del Plata. Luego de 6 meses los paneles se retiraron y observaron en el laboratorio bajo lupa binocular y microscopio óptico. La estimación de la fijación para cada especie sobre los paneles se evaluó con una grilla de 25 puntos al azar. Se trabajó por triplicado, con controles y se aplicaron test estadísticos.

Las pinturas con cobre+timol presentaron una muy buena performance dado que disminuyeron el asentamiento del biofouling. No se registraron diferencias significativas en los porcentajes de cobertura entre las formulaciones con cobre y la pintura con cobre+timol ( $>>0,05)$. El timol empleado como aditivo en la formulación antiincrustante permitió reducir $90 \%$ el contenido de cobre manteniendo la efectividad en servicio.

Palabras clave: incrustaciones biológicas, timol, óxido cuproso, pinturas antiincrustantes

\section{ABSTRACT}

Biofouling leads to deterioration of any submerged material. The most widespread method for control is the application of cuprous oxide antifouling paints which are toxic. For this reason non-toxic natural products could be combined with small amounts of copper to diminish its contribution to the environment.

Thymol is isolated from essential oils of several plants (thyme, oregano, basil) and it is well known for its antimicrobial properties. The hypothesis proposed in this study is this compound would inhibit initial steps in fouling sequence and consequently would also inhibit settlement of later species.

The aim of this work was to reduce the amount of copper in antifouling paints employing thymol as an additive. Two antifouling paints were prepared, one containing $16 \% \mathrm{v} / \mathrm{v}$ copper and another with $1.6 \%$ copper $+2 \%$ thymol. Paints were applied on acrylic panels and were submerged in Mar del Plata harbor.

After 6 months of immersion in Mar del Plata harbor fouling cover percentage on panels was estimated under stereomicroscope and optic microscope. Estimation of fouling cover by species was carried out using a 25 random point grid. Also, control paints and unpainted acrylic tiles were simultaneously submerged. All tests were performed in triplicate.

Paints containing $1.6 \%$ copper + thymol and $16 \%$ copper were effective. No significance differences were registered in cover percentage between copper-based paints and copper+thymol based paints $(\mathrm{p}>0.05)$. 
Although these formulations showed a similar performance, copper+thymol based paint contains $90 \%$ lesser copper than a traditional copper based formulation. Then, the aim of this work was reached.

Keywords: biofouling, thymol, cuprous oxide, antifouling paints

\section{INTRODUCCIÓN}

La aplicación de pinturas antifouling es el método más empleado para controlar el biofouling marino. Estas pinturas contienen sustancias tóxicas tales como el tributil estaño (TBT) y óxido cuproso que son altamente contaminantes del agua, los sedimentos y la biota. Si bien el uso del TBT ha sido prohibido debido al daño que ocasiona en el ecosistema marino [1], no ha sucedido lo mismo con el óxido cuproso. En este contexto se buscan productos de origen natural que permitan reducir el contenido de cobre de las formulaciones y de este modo disminuir su aporte al medio ambiente.

Los aceites esenciales extraídos de plantas tienen aplicaciones en la medicina étnica y preservación de alimentos así como en la industria cosmética y farmacéutica [2-4]. Si bien estos compuestos son de origen natural, muchos de ellos se obtienen por síntesis en laboratorio lo que representa una ventaja desde el punto de vista de su disponibilidad en el mercado. En este sentido se eligió al timol a fin de evaluar su comportamiento en laboratorio y en el mar. La hipótesis que se plantea es que podría actuar inhibiendo el proceso inicial de asentamiento del biofouling marino y consecuentemente evitar la fijación posterior del macrofouling.

El timol (Figura 1) se aísla de los aceites esenciales de numerosas plantas como el tomillo, orégano, mejorana y albahaca. Estas plantas son utilizadas como condimentos en numerosos alimentos y son reconocidas como seguras por la FDA de Estados Ubidos (GRAS: generally recognized as a safe, Food and Drug Administration). Son numerosas las propiedades descriptas para el timol; en trabajos previos se comprobó que posee actividad antibacteriana, antifúngica, antihelmíntica y antioxidante, propiedades que están relacionadas con su alto contenido fenólico [ㄷ-]. En otras líneas de investigación se demostró también que este compuesto reduce el contenido de patógenos, olores y emisión de gases del efecto invernadero en granjas de cría de porcinos [10]. Por otra parte, el timol presentó un alto poder insecticida dado que vapores de aceites esenciales de vegetales ricos en el compuesto resultaron eficaces contra huevos, larvas y adultos del coleóptero Callosobranchus maculatus ("gorgojo del frijol”) y de Spodoptera litura ("gusano gris del tabaco"). Asimismo, resultó efectivo como acaricida (escabicida) y como larvicida en mosquitos vectores de enfermedades endémicas como malaria y paludismo y tisanópteros plaga de plantaciones de ajo, cebolla, lino, maní, papa, tomate, pimiento, vid y zanahoria [11-17].<smiles>Cc1ccc(C(C)C)c(O)c1</smiles>

Figura 1: Estructura del timol

Es importante destacar que el timol es un compuesto que puede sufrir distintos procesos de biotransformación en el medio ambiente por medio de una amplia variedad de organismos, entre ellos, bacterias, hongos y hasta plantas superiores [18-21]. Chamberlain y Dagley [18] demostraron que Pseudomonas putida es capaz de degradar al timol en ácidos carboxílicos y cetoácidos sencillos a través de una ruta metabólica que involucra a varios metabolitos intermediarios, entre ellos anillos bencénicos di y trihidroxilados y con estructura quinoide. Hahn y colaboradores [19] han reportado que bacterias Grampositivas de los géneros Nocardia y Mycobacterium degradan al timol mediante dos diferentes mecanismos oxidativos y que los productos obtenidos son menos tóxicos que el propio timol. Por otra parte, se ha encontrado que varios géneros de hongos, en particular Colletotrichum acutatum y Botryodiplodia theobromae también degradan al timol dando una compleja mezcla de productos de degradación [20]. El timol es usado comercialmente en colmenas para controlar al ácaro Varroa destructor [22] y también se lo ha 
utilizado como biofumigante en plantaciones de tomate para el control de microorganismos [23]. Se ha determinado que cuando el timol es usado como fumigante se incorpora naturalmente a los ciclos biogeoquímicos (disipación, mineralización y biodegradación) y los residuos ligados al suelo o agua son insignificantes [24].

Los objetivos de este trabajo son, por un lado, evaluar la potencial actividad antifouling del timol en el laboratorio y por otro reducir el contenido de cobre en las pinturas antifouling usándolo como aditivo.

\section{MATERIALES Y MÉTODOS}

\subsection{Bioensayos}

Los ensayos se realizaron sobre larvas nauplii y cypris de Balanus amphitrite, organismo con exoesqueleto calcáreo considerado entre los más perjudiciales dentro de la comunidad incrustante.

Se expusieron 30 nauplii II de Balanus amphitrite a distintas soluciones de timol durante 24 horas a fin de determinar la concentración necesaria para inhibir el $50 \%$ de la población $\left(\mathrm{LC}_{50}\right)$. Los parámetros estudiados para evaluar el efecto del compuesto fueron los movimientos de natación y el fototactismo.

Por otra parte se realizaron bioensayos de asentamiento utilizando 20 larvas cypris que fueron mantenidas a $6^{\circ} \mathrm{C}$ durante 4 días previo al ensayo [25]. Se determinó la concentración efectiva para inhibir el asentamiento del $50 \%$ de la población $\left(\mathrm{EC}_{50}\right)$. Las larvas no nadadoras, con apéndices extendidos o con sus valvas cerradas fueron consideradas como inactivas. La observación se realizó bajo estereomicroscopio y se registraron los porcentajes de larvas nadadoras, inactivas y fijadas; los valores de $\mathrm{LC}_{50}$ y $\mathrm{EC}_{50}$ fueron determinados por análisis Probit.

Se evaluó el efecto de soluciones de timol en el rango de concentración de 2-60 $\mu \mathrm{M}$ y 2-6 $\mu \mathrm{M}$ para los test de toxicidad y asentamiento, respectivamente. En todos los casos para completar los estudios se llevaron a cabo pruebas de recuperación trasladando los organismos a agua de mar limpia. Las experiencias se realizaron a $22 \pm 2^{\circ} \mathrm{C}$, por triplicado con sus respectivos controles; se aplicaron test estadísticos evaluándose los resultados con ANOVA y test de contraste Tukey.

\subsection{Ensayos con pinturas en el mar}

Se prepararon dos pinturas antifouling, una conteniendo $16 \% \mathrm{v} / \mathrm{v}$ de cobre (PC) y otra con $1,6 \%$ de cobre $+2 \%$ de timol ( $\mathrm{Cu}+$ timol) (Tabla 1). El PVC (concentración de pigmento en volumen) es la relación en volumen de pigmento que éste ocupa con respecto al volumen total de pintura seca (pigmentos + resina). El PVC debe mantenerse constante para no introducir una nueva variable al sistema de estudio. Por lo tanto la reducción del contenido de óxido cuproso en la formulación debe ir acompañado con el aumento en la concentración de los otros pigmentos de la formulación, en este caso la tiza. El vehículo de la pintura (resina + solvente) se preparó en una dispersora de alta velocidad agregando lentamente la resina colofonia a la mezcla de solventes. Luego, las pinturas se prepararon en un molino de bolas de 11 de capacidad dispersando los pigmentos y los demás componentes en el vehículo durante 24 horas. Las pinturas fueron aplicadas con pincel hasta un espesor final de película seca de $100 \pm 5 \mu \mathrm{m}$, sobre paneles de acrílico $(8 \mathrm{~cm} \times 12 \mathrm{~cm})$ previamente arenados y desengrasados con tolueno. Los paneles pintados y los controles sin pintura (AC) se expusieron en el Club de Motonáutica del puerto de Mar del Plata (38 $\left.08^{\prime} \mathrm{S}-57^{\circ} 31^{\prime} \mathrm{W}\right)$ a $50 \mathrm{~cm}$ por debajo de la superficie del agua.

Tabla 1: Composición de las pinturas expresadas en porcentaje en volumen

\begin{tabular}{lcc}
\hline Componentes & PC & Cu+timol \\
\hline Óxido cuproso & 16,0 & 1,6 \\
Tiza & 11,0 & 23,4 \\
Timol & ---- & 2,0 \\
Colofonia & 27,0 & 27,0 \\
Ácido oleico & 6,0 & 6,0 \\
\hline Xileno/aguarrás mineral & 40,0 & 40,0 \\
\hline
\end{tabular}


Luego de 6 meses de inmersión los paneles se retiraron y se evaluó la actividad antiincrustante de las pinturas por medio de la estimación de los porcentajes de cobertura total de todas las especies fijadas. Los paneles se observaron en el laboratorio bajo lupa binocular y microscopio óptico. Las experiencias se realizaron por triplicado. Se aplicaron test estadísticos evaluándose los resultados con ANOVA y test de contraste Tukey.

\section{RESULTADOS Y DISCUSIÓN}

\subsection{Bioensayos}

Los ensayos de toxicidad realizados con nauplii de Balanus amphitrite en el laboratorio demostraron un marcado efecto inhibitorio del timol con un $\mathrm{LC}_{50}$ de $4,41 \mu \mathrm{M}$ para las 24 horas de exposición (Figura 2). Todos los organismos expuestos a las distintas concentraciones de timol recuperaron su actividad normal cuando fueron transferidos a agua limpia, por lo que se infiere que el efecto inhibidor es temporal.

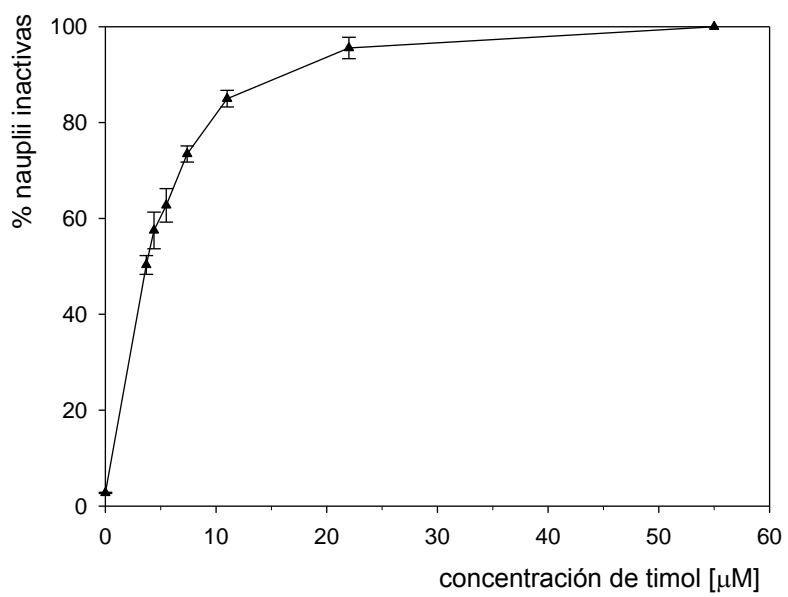

Figura 2: Ensayo de toxicidad de 24 horas para Balanus amphitrite en soluciones de timol.

En cuanto al test de asentamiento, el análisis ANOVA indica que hay diferencias significativas en los porcentajes de fijación de las cypris frente a las distintas concentraciones ensayadas $(\mathrm{p}<0,05)$. Por otra parte, la concentración inhibitoria para el $50 \%$ de la población de cypris $\left(\mathrm{EC}_{50}\right)$ fue de 2,26 $\mu \mathrm{M}$ (Figura 3). De un modo similar a lo observado para las nauplii, las cypris se recuperaron una vez trasladadas a agua de mar.

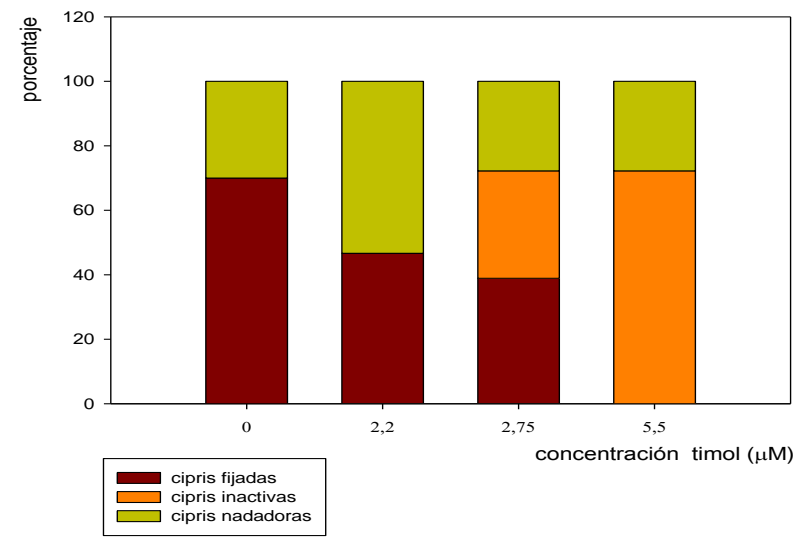

Figura 3: Test de asentamiento de 24 horas para B. amphitrite en soluciones de timol.

Los valores que resultaron efectivos en los bioensayos confirman no sólo las propiedades antifouling del timol sino también que actúa en muy bajas concentraciones. En estudios realizados por otros autores empleando timol las concentraciones efectivas sobre bacterias y hongos fueron mayores en dos y tres órdenes de magnitud [26- 33]. 
El índice terapéutico [34] es una medida que expresa la efectividad de un compuesto en relación a su toxicidad y se calcula como $\mathrm{LC}_{50} / \mathrm{EC}_{50}$. Para los experimentos con timol fue de 1,95. Dado que este valor es mayor que 1 , se concluye que el timol actúa por un mecanismo de acción no tóxico, lo cual esta de acuerdo con los resultados de los ensayos de recuperación de las larvas. Los dos estadios larvales recuperaron completamente su actividad cuando dejan de estar en contacto con el timol. Si bien existe un acuerdo generalizado de que el timol, por su carácter lipofílico, produce una desorganización de las membranas celulares en bacterias [ $\underline{35}, \underline{36}$ ], no se conoce aún cual es el mecanismo de acción involucrado en los organismos incrustantes estudiados en este trabajo.

\subsection{Ensayos con pinturas en el mar}

Como puede observarse en la Figuras 4, 5 y 6, luego de 6 meses de exposición en el puerto de Mar del Plata, las pinturas con cobre+timol fueron tan efectivas como la que contienen cobre al $16 \%$ dado que presentaron diferencias significativas en la fijación de macro y microfouling respecto a los controles de acrílico $(\mathrm{p}<0.05)$.

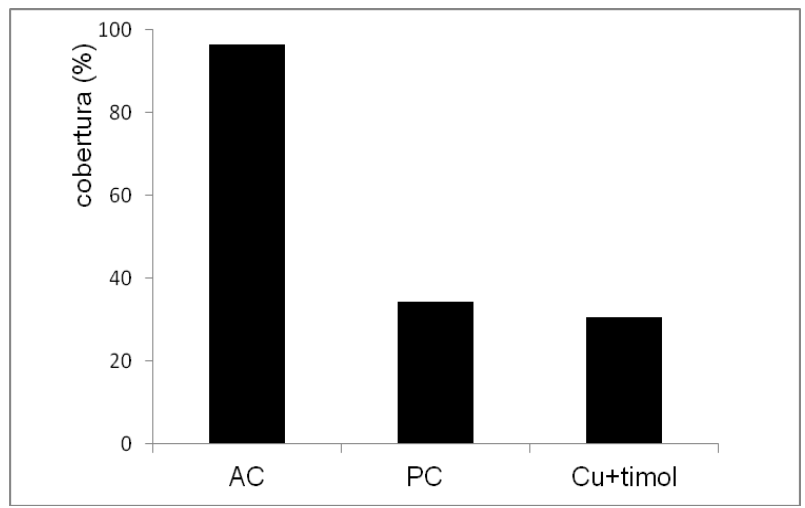

Figura 4: Macrofouling: cobertura porcentual de los diferentes tratamientos expuestos en el puerto de Mar del Plata luego de 6 meses de inmersión. AC: control sin pintura; PC: pintura con 16\% v/v de cobre; Cu+timol: pintura con $1,6 \% \mathrm{v} / \mathrm{v}$ de cobre $+2 \%$ de timol

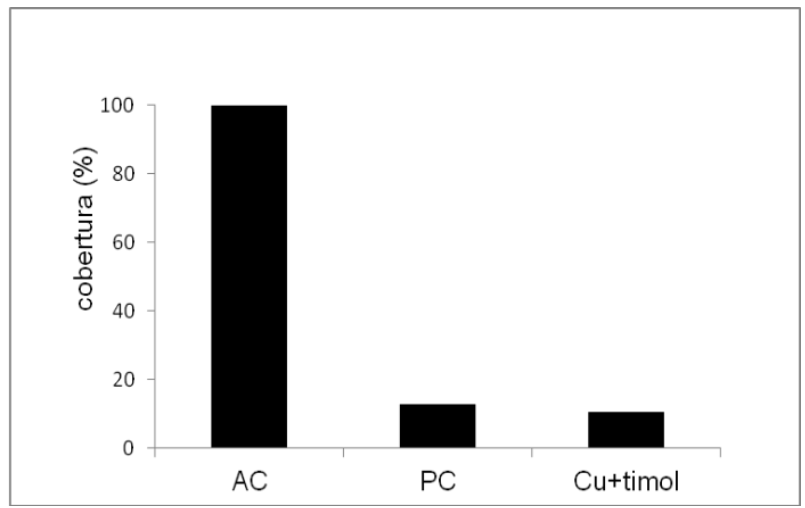

Figura 5: Microfouling: cobertura porcentual de los diferentes tratamientos expuestos en el puerto de Mar del Plata luego de 6 meses de inmersión. AC: control sin pintura; PC: pintura con 16\% v/v de cobre; $\mathrm{Cu}+$ timol: pintura con 1,6\%v/v de cobre $+2 \%$ de timol 


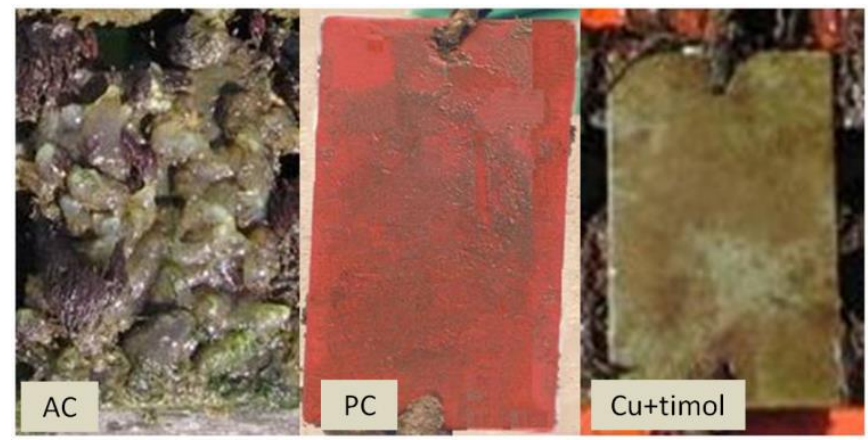

Figura 6: Paneles expuestos en el puerto de Mar del Plata luego de 6 meses de inmersión. AC: control sin pintura; PC: pintura con $16 \% \mathrm{v} / \mathrm{v}$ de cobre; $\mathrm{Cu}+$ timol: pintura con $1,6 \% \mathrm{v} / \mathrm{v}$ de cobre $+2 \%$ de timol

Los experimentos realizados indican que el timol presenta poder antiincrustante, una nueva actividad que se agrega a la gran variedad de propiedades conocidas previamente. Por otra parte, es de destacar su efecto temporario que resulta muy promisorio desde un punto de vista ecológico, dado que no interfiere en la continuidad del ciclo de vida de los organismos. En un sentido amplio, la utilización de timol en el campo de los recubrimientos ya había sido propuesta en la fabricación de films para protección de alimentos a fin de evitar la degradación y prolongar, de este modo, la vida útil del producto [므].

Indudablemente, las pinturas tradicionales conteniendo óxido cuproso presentan una muy buena performance en el mar. Sin embargo, la reducción en la liberación de metales pesados en el mar es una tendencia creciente que impulsa la búsqueda de variantes menos contaminantes e igualmente efectivas.

La disminución del contenido de cobre en pinturas antiincrustantes fue abordada previamente con resultados promisorios utilizando pigmentos con bajo contenido de cobre sintetizados en el laboratorio. En este sentido se elaboraron formulaciones con una importante reducción en el contenido de cobre basadas en la deposición de tiocianato cuproso sobre un núcleo central de distintos materiales inertes: carbonato de calcio, cuarzo micronizado, óxido férrico y óxido de cinc, son los denominados "core-shell pigments". Las pinturas elaboradas con estos pigmentos resultaron efectivas conteniendo una cantidad de tóxico 35 veces menor que en las formulaciones convencionales a base de óxido cuproso [37]. Por otro lado, se sintetizó un pigmento a base de tanato cúprico y se comprobó que presenta actividad antifouling en el mar con una reducción del contenido de cobre del orden de 40 veces respecto de una pintura convencional a base de óxido cuproso [38]. Peres y colaboradores [39] han trabajado también en este tema y han logrado una muy buena performance en servicio utilizando dodecanoato de cobre como pigmento antiincrustante. Aunque en tales casos la reducción del contenido de cobre fue mayor, el proceso industrial para preparar estos pigmentos antiincrustantes implica una gran inversión. Por el contrario, nuestros esfuerzos están enfocados a la obtención de nuevas formulaciones de pintura antifouling que impliquen el uso de materias primas económicas y disponibles en el mercado, tales como el timol. Los resultados de este trabajo demuestran que la combinación de bajas cantidades de cobre con timol resultó efectiva y promisoria en virtud de su posible transferencia a la industria de pinturas marinas.

\section{CONCLUSIÓN}

Los resultados de laboratorio indican que el timol es un potente inhibidor de las larvas nauplii y cypris de Balanus amphitrite y actúa a bajas concentraciones a través de un mecanismo no tóxico. La efectividad de las pinturas formuladas con timol $+1,6 \%$ de cobre fue similar a las pinturas que contenían $16 \%$ de cobre como pigmento antifouling. Es de destacar que si bien presentan una performance equivalente, la primera tiene un contenido de cobre $90 \%$ menor, con lo cual se satisface el objetivo propuesto de reducir la cantidad de cobre manteniendo la actividad antifouling.

\section{AGRADECIMIENTOS}

Los autores agradecen al CONICET, CICPBA y Universidad Nacional de la Plata por el apoyo brindado para realizar este trabajo. 


\section{BIBLIOGRAFÍA}

[1] CHAMBERS, L., STOKES, K., WALSH, F., et al.,"Modern approaches to marine antifouling coatings", Surface and Coatings Technolgy, v. 201, pp. 3642-3652, 2006.

[2] PASCUAL, M., SlOWING, K., CARRETERO, E., et al.,"Lippia: traditional uses, chemistry and pharmacology: a review”, Journal of Ethnopharmacology, v. 76, pp. 201-214, 2001.

[3] BURÓ, S., "Essential oils: their antibacterial properties and potential applications in foods-a review", International Journal of Food Microbiology, v. 94, pp. 223- 253, 2004.

[4] EDRIS, A., "Pharmaceutical and therapeutic potentials of essential oils and their individual volatile constituents: a review", Phytotherapy Research, v. 21, pp. 308-323, 2007.

[5] ROTA, M., HERRERA, A., MARTÍNEZ, R., et al.,"Antimicrobial activity and chemical composition of Thymus vulgaris, Thymus zygis and Thymus hyemalis essential oils”, Food Control, v. 19, pp. 681-687, 2008.

[6] KORDALI, S., CAKIR, A., OZER, H., et al.,"Antifungal, phytotoxic and insecticidal properties of essential oil isolated from Turkish Origanum acutidens and its three components, carvacrol, thymol and pcymene", Bioresource Technology, v. 99, pp. 8788-8795, 2008.

[7] VASCONCELOS, A., BEVILAQUA, C., MORAIS, S., et al.,"Anthelmintic activity of Croton zehntneri and Lippia sidoides essential oils”, Veterinary Parasitology, v. 148, pp. 288-294, 2007.

[8] TEPE, B., SOKMEN, M., ASKIN AKPULAT, H., et al., "Antioxidative activity of the essential oils of Thymus sipyleus subsp. sipyleus var. sipyleus and Thymus sipyleus subsp. sipyleus var. rosulans", Journal of Food Engineering, v. 66, pp. 447-454, 2005.

[9] DIMITRIJEVIC, S., MIHAJLOVSKI, K., ANTONOVIC, D., et al., "A study of the synergistic antilisterial effect of a sub-lethal dose of lactic-acid and essential oils from Thymus vulgaris L., Rosmarinus officinalis L. and Origanum vulgare L.”, Food Chemistry, v. 104, pp. 774-782, 2007.

[10] VAREL, V., WELLS, J., "Influence of thymol and urease inhibitor on coliform bacteria, odor, urea, and methane from a swine production manure pit", Journal of Environmental Quality, v. 36, pp. 773-779, 2007.

[11] AMER, A., MEHLHORN, H., "Larvicidal effects of variuos essential oils against Aedes, Anopheles, and Culex larvae (Diptera, Culicidae)", Parasitology Research, v. 99, pp. 466-472, 2006.

[12] GLEISER, R., ZYGADIO, J., "Insecticidal properties of essential oils from Lippia turbinata and Lippia polystuchya (Verbenaceae) against Culex quinquefasciatus (Diptera: Culicidae), Parasitology Research, v. 101, pp. 1349-1354.

[13] ISMAN, M., WAN, A., PASSREITER, C., "Insecticidal activity of essential oils to the tobacco cutworm, Spodoptera litura", Fitoterapia, v. 72, pp. 65-68, 2001.

[14] KNIO, K., USTA, J., DAGHER, S., et al., "Larvicidal activity of essential oils extracted from commonly used herbs in Lebanon against the seaside mosquito, Ochlerotatus caspius", Bioresource Technology, v. 99, pp. 763-768, 2008.

[15] SAHAF, B., MOHARRAMIPOUR, S., "Fumigant toxicity of Carum copticum and Vitex pseudonegundo essential oils against eggs, larvae and adults of Callosobruchus maculatus", Journal of Pest Science, v. 81, pp. 213-220, 2008.

[16] SEDY, K., KOSCHIER, E., "Bioactivity of carvacrol and thymol against Frankliniella occidentalis and Thrips tabaci”, Journal of Applied Entomology, v. 127, pp. 313-316, 2003.

[17] ARDESHIR, A., EBADI, R., TAHMASEBI, G., "Laboratory evaluation of some plant essence to control Varroa destructor (Acari: Varroide)", Experimental and Applied Acarology, v. 27, pp. 319-327, 2002.

[18] CHAMBERLAIN, E., DAGLEY, S.,"The metabolism of thymol by a Pseudomonas", Biochemical Journal, v. 110, pp.755-763, 1968.

[19] HAHN, V., SÜNWOLDT, K., MIKOLASCH, A., et al., "Two different primary oxidation mechanisms during biotransformation of thymol by gram-positive bacteria of the genera Nocardia and Mycobacterium", Applied Microbiology and Biotechnology, v. 97, pp. 1289-1297, 2013.

[20] NUMPAQUE M., OVIEDO, L., GIL, J., et al., "Thymol and carvacrol: biotransformation and antifungal activity against the plant pathogenic fungi Colletotrichum acutatum and Botryodiplodia theobromae". Tropical Plant Pathology, v. 36, pp. 003-013, 2011.

[21] SHIMODA, K., KONDO, Y., NISHIDA, T., et al., "Biotransformation of thymol, carvacrol, and eugenol by cultured cells of Eucalyptus perriniana", Phytochemistry, v. 67, pp. 2256-2261, 2006. 
[22] AHMAD, K., RAZZAQ, A., ABBASI, K., et al., "Thymol as control agent of mites (Varroa destructor) on honeybees (Apis mellifera)", Pakistan Journal of Agricultural Research, v. 26, pp. 316-320, 2013.

[23] JI, P., MOMOL, M., OLSON, S., et al., "Evaluation of thymol as biofumigant for control of bacterial wilt of tomato under field conditions", Plant Disease, v. 89, pp. 497-500, 2005.

[24] HU, D., COATS, J., "Evaluation of the environmental fate of thymol and phenethyl propionate in the laboratory", Pest Management Science, v. 64, pp. 775-779, 2008.

[25] RITTSCHOF, D., CLARE, A., GERHART, D., et al., "Barnacle in vitro assays for biologically active substances: toxicity and settlement assays using mass cultured Balanus amphitrite amphitrite Darwin", Biofouling, v. 6, pp. 115-122, 1992.

[26] DEL NÓBILE, M., CONTE, A., INCORONATO, A., et al., "Antimicrobial efficay and release kinetics of thymol from zein films", Journal of Food Engineering, v. 89, pp. 57-63, 2008.

[27] BENDAHOU, M., MUSELLI, A., GRIGNON-DUBOIS, M., et al., "Antimicrobial activity and chemical composition of Origanum glandulosus Desf. Essential oil and extract obtained by microwave extraction: comparison with hydrodistillation”, Food Chemistry, v. 106, pp. 132-139, 2008.

[28] BRAGA, P., CULICI, M., ALFIERI, M., et al., "Thymol inhibits Candida albicans biofilm formation and mature biofilm", International Journal of Antimicrobial Agents, v. 31, pp. 472-477, 2008.

[29] DAFERERA, D., ZIOGAS, B., POLISSIOU, M., "The effectiveness of plant essential oils on the growth of Botrytis cinerea, Fusarium sp. and Clavibacter michiganesis subsp. michiganensis", Crop Protection, v. 22, pp. 39-44, 2003.

[30] YAMAZAKI, K., YAMAMOTO, T. KAWAI, Y., et al., "Enhancement of antilisterial activity of essential oil constituents by nisin and diglycerol fatty acid ester", Food Microbiology, 21, pp. 283-289, 2004.

[31] VALERO, M. y GINER, M., "Effects of antimicrobial components of essential oils on growth of Bacillus cereus INRA L2104 in and the sensory qualities of carrot broth", International Journal of Food Microbiology, v. 106, pp. 90-94, 2006.

[32] AMIN, G., SOURMAGHI, S., ZAHEDI, M., et al., "Essential oil composition and antimicrobial activity of Oliveria decumbens", Fitoterapia, 76, pp. 704-707, 2005.

[33] DELGADO, B., FERNÁNDEZ, P., PALOP, A., et al., "Effect of thymol and cymene on Bacillus cereus vegetative cells evaluated through the use of frequency distributions", Food Microbiology, v. 21, pp. 327334, 2004.

[34] RITTSCHOF, D., SASIKUMAR, N., MURLLESS, D., et al., "Mixture interactions of lactones and furans and a commercial biocide: toxicity and antibarnacle settlement activity", In: Recent developments in biofouling control, Thompson, M. F., Nagabhushanam, R., Sarojini, R., Fingerman, M., editors, Rotterdam, Balkema AA, pp. 269-274, 1994.

[35] WALSH S., MAILlARD, J., RUSSELL, A., et al., "Activity and mechanisms of action of selected biocidal agents on Gram-positive and -negative bacteria", Journal of Applied Microbiology, v. 94, 240-247, 2003.

[36] SHAPIRO, S., GUGGENHEIM, B., "The action of thymol on oral bacteria", Oral Microbiology and Immunology, 10, pp. 241-246. 1995.

[37] PÉREZ, M., GARCÍA, M., DEL AMO, B., BLUSTEIN, G., et al., "Core-shell pigments in antifouling paints", Surface Coatings International Part B, Coatings Transactions, v. 86, pp. 259-262, 2003.

[38] PÉREZ, M., BLUSTEIN, G., GARCÍA, M., et al., "Cupric tannate: a low copper content antifouling pigment", Progress in Organic Coatings, v. 55, pp. 311-315, 2006.

[39] PERES, R., BALDISSERA, A., ARMELIN, E., et al., "Marine-friendly antifouling coating based on the use of a fatty acid derivative as a pigment", Materials Research, v. 17, pp. 720-727, 2014. 